W.D.R. Writer $\mathrm{MB}$ CH B $\mathrm{FRCP}(\mathrm{C})$, Jan M. Davies MSC MD FRCP(C), Leo Strunin MD FFARCS FRCP(C)

\title{
Editorial
}

\section{Trial by media: the bupivacaine story}

When it appears a drug may cause serious side effects, physicians deserve careful presentation of the evidence. This is particularly true when a drug has been in use for several years without apparent ill effect. The recent controversy over the use of 0.75 per cent bupivacaine in obstetric anaesthesia draws attention again to the way in which side effects of drugs are investigated. On August 22, 1983, the United States Food and Drug Administration (F.D.A.) sent a letter to all American doctors recommending that 0.75 per cent bupivacaine not be used for obstetric anaesthesia because of reports of cardiac arrest with this concentration of the drug. Quickly the matter spread to the Canadian media which conducted interviews with "anaesthesia experts," not all of whom were obstetric anaesthetists. Then, the Drugs Directorate of the Health Protection Branch (H.P.B.) of the Department of National Health and Welfare Ottawa (the Canadian equivalent of the F.D.A.), after apparently limited consultation, asked the Canadian manufacturer of bupivacaine to warn Canadian doctors and to recommend reduced doses of the drug during childbirth. Thus, on August 29, 1983, Winthrop Laboratories sent a letter to all doctors in Canada stating that 0.75 per cent bupivacaine not be used for obstetrics and that caution be exercised with its use in nonpregnant patients. In addition, the letter advised that bupivacaine no longer be used for abstetrical paracervical anaesthesia and that bupivacaine is not recommended nor approved for use in intravenous regional anaesthesia.

Questions concerning cardiotoxicity of bupivacaine were first raised by Dr. G.A. Albright, Asso-

From the Departments of Anaesthesia and Obstetrics and Gynaecology, Dalhousie University, Halifax, Nova Scotia, and the Department of Anaesthesia, Foothills Hospital, at the University of Calgary, Calgary, Alberta. ciate Professor, Stanford University, California, who specialises in obstetric anaesthesia. In a 1979 editorial ${ }^{1}$ he reported six cases of sudden cardiovascular collapse, immediately after presumed intravascular injection of bupivacaine or etidocaine, despite a negative aspiration test. He postulated that these agents, in contrast to lidocaine, could cause almost simultaneous seizures and cardiovascular collapse and stated cardiopulmonary resuscitation might be difficult, if not impossible. By 1981, Dr. Albright had gathered additional anecdotal reports of bupivacaine cardiotoxicity and submitted these as a paper for publication in journals specialising in anaesthesia and obstetrics and gynaecology; the manuscript, despite revision, was not accepted. Subsequently, Dr. Albright has presented ${ }^{2}$ a further "tally" of 15 maternal deaths in the United States since 1973, after extradural injection of bupivacaine, with doses ranging from 75 to $16.5 \mathrm{mg}$. However, this report is difficult to interpret since it is in summary form. Personal communication with Dr. Albright reveals he has presented a detailed account of his current information (36 maternal and 20 non-obstetric cases) to the F.D.A. at a recent inquiry into toxicity of local anaesthetic agents.

Other clinical reports $s^{3,4}$ have described convulsions, associated with hypoxia and acidosis, from presumed intravascular injection of bupivacaine in non-obstetric subjects; however, these patients responded to immediate resuscitation with 100 per cent oxygen. While some animal studies of bupivacaine have revealed an arrhythmogenic and apparent cardiotoxic effect, ${ }^{5-8}$ perhaps enhanced by acidosis and hypoxia, ${ }^{9,10}$ other laboratory research does not support bupivacaine cardiotoxicity. ${ }^{11-13}$ Species, ${ }^{14}$ dosage and protocol differences make interpretation of these findings difficult.

Since all experimental work on bupivacaine cardiotoxicity is in animals, there is not direct 
support for Dr. Albright's view that there is increased cardiac susceptibility to bupivacaine in the parturient; however, there is good reason to believe any local anaesthesia, if injected accidentally into the distended extradural-azygos venous system, will reach the heart in significant concentration. ${ }^{15}$ Therefore, careful epidural technique demands firstly, the use of a suitable test dose; secondly, slow injection of the drug in divided and appropriate doses; and thirdly, repeated checking to exclude intravascular administration. One negative aspiration test is inadequate, since intravascular local anaesthetic, at any time, may cause convulsions and cardiac depression.

In Canada, to date, there have been no reports of maternal deaths associated with bupivacaine; there has been one death reported to the H.P.B. associated with the use of 0.75 per cent bupivacaine for intercostal nerve block in a non-pregnant patient. In the United Kingdom, where all maternal deaths are reviewed ${ }^{16}$ confidentially, no death directly attributable to 0.75 per cent bupivacaine has yet been reported; however. deaths in non-pregnant patients associated with intravenous regional analgesia using bupivacaine have occurred. ${ }^{17}$ It seems, therefore, that although bupivacaine may be toxic when injected intravascularly, maternal mortality has been confined to the United States. This may reflect usage of the drug, competence of the individual administering the drug, or some other factor yet to be determined.

How may Canadian anaesthetists learn from this episode? Bupivacaine 0.75 per cent, used without ill effect for obstetric anaesthesia in Canada since 1975, has been withdrawn, essentially on anecdotal evidence from a foreign country. Although Dr. Albright and the government drug regulatory bodies of the United States and Canada may prove to be correct, a significant opportunity has been lost in Canada to evaluate properly the safety and clinical role of 0.75 per cent bupivacaine for obstetric anaesthesia. No ethics committee now would likely give permission for such an investigation; trial by media has removed this possibility.

Perhaps it is time for the Canadian Anaesthetists' Society to consider how it should advise the H.P.B. and pharmaceutical companies when questions concerning anaesthetic drugs are raised. This would allow individual anaesthetists a reference source rather than finding themselves cornered by the media and forced to issue ex cathedra statements open to misinterpretation. In addition, pressure should be brought to bear by all anaesthetists on the H.P.B. to take the advice of the Canadian Anaesthetists' Society rather than to allow things to happen by negative serendipity.

\section{Quand les medias se font juges: le cas de la bupivacaine}

Lorsqu'il y a lieu de croire qu'un médicament possède des effets secondaires graves, il est essentiel de mettre au courant les médecins eux-mêmes. Ceci est particulièrement important dans le cas d'un médicament utilisé depuis plusieurs annees sans conséquences nefastes évidentes. La controverse récente sur l'utilisation de la bupivacaine à 0.75 pour cent pour anesthésie régionale en obstétrique, nous montre la façon dont les effets secondaires des médicaments sont étudiés.

En 1983 l'Agence americaine des aliments et des drogues (FDA) écrivit à tous les médecins américains leur recommandant de ne pas se servir de la bupivacaine à 0.75 pour cent en anesthésie obstérricale suite à des rapports d'arrêt cardiaque après utilisation de cette concentration de la drogue.

Les médias canadiens s'emparèrent vite de l'affaire, et interviewèrent des "experts en anesthésie" qui n'étaient pas tous des anesthésistes obstétricaux. C'est apparemment dans cette foulée que la division de la Protection de la Santé et Bien-Etre Canada (équivalent canadien de la FDA) a demandé aux fabricants de la bupivacaine de faire une mise au point sur ce sujet et de recommander des doses réduites durant l'accouchernent. C'est ainsi que le 29 août 1983 les laboratoires Winthrop ont émis une directive recommandant d'éviter la bupivacaïne à 0.75 pour cent en obstétrique et de n'utiliser cette concentration qu'avec prudence chez les patientes non enceintes. De plus, la lettre recommandait de discontinuer l'utilisation de la bupivacaüne lors de l'anesthésie obstétricale para- 
cervicale et de ne pas l'utiliser ni l'approuver pour utilisation lors d'une anesthésie régionale intraveineuse.

Le docteur G.A. Albright, professeur adjoint de l'université de Stanford en Californie et spécialiste en anesthésie obstétricale, a été le premier à se poser des questions au sujet de la cardiotoxicité de la bupivacainne. En $1979^{\mathrm{L}}$ dans une page éditoriale d'Anesthesiology, il discutait de six cas de collapsus cardiovasculaire à la suite d'emploi de bupivacaïne ou d'étidocaïne. Ces collapsus sont survenus à la suite d'une injection que l'on croit intra-vasculaire en dépit d'un test d'aspiration nćgatif. Il en inférait que, contrairement à la lidocaïne, ces agents pouvaient causer des convulsions et un collapsus cardiovasculaire instantanés rendant, de ce fait, la ressuscitation cardiopulmonaire difficile, sinon impossible.

En 1981, le docteur Albright ayant accumulé d'autres éléments anecdotiques en rapport avec la cardiotoxicité de la bupivacaine prépare un travail qu'il soumet pour publication dans les revues spécialisées en anesthésie, et en obstétrique et gynécologie; le manuscrit est rejeté même à la suite de revision et de corrections. Ultérieurement, le docteur Albright présente une liste descriptive de 15 décès à l'accouchement survenus aux Etats-Unis ${ }^{2}$ depuis 1973 après des injections d'extradurales de bupivacaïne à la dose de 75 à $165 \mathrm{mg}$. Cependant, le format de ce rapport le rend difficile à interpréter. Au cours de conversations privées, le docteur Albright nous a souligné qu'il a été invité par la FDA à présenter un rapport détaille de ses informations portant sur 56 cas dont 36 en ancsthésic obstétricale.

D'autres études ${ }^{3,4}$ hors du contexte obstétrical ont rapporté des convulsions reliées à une hypoxie et une acidose à la suite d'une injection qu'on présume intravasculaire, de bupivacaïne; cependant, ces malades ont été rapidement réanimés au moyen d' $\mathrm{O}_{2}$ pur.

Certaines ćtudes en laboratoire ont décelé des propriétés arythmogéniques ${ }^{5.8}$ et cardiotoxiques de la bupivacaïne chez l'animal, toxicité amplifiée par l'acidose et l'hypoxie; 9,10 cependant, d'autres travaux en laboratoire n'arrivent pas aux mêmes conclusions. ${ }^{11-13}$ Des différences d'espèces, ${ }^{14}$ de posologies et de protocole expérimental rendent difficile l'interprétation de ces résultats.

Les travaux expérimentaux sur la bupivacaïne étant faits sur des animaux, aucun n'appuie de façon directe l'opinion du docteur Albright à l'effet que la cardiotoxicité de la bupivacaine est augmentée chez la parturiente. Cependant, on a raison de croire que n'importe quel anesthésique local injecté accidentellement dans le réseau veineux extradural azigos parviendra au cceur en concentration importante. $^{15}$ Par conséquent, l'administration d'un agent par voie péridurale exige une technique très précisément planifiée: 1) l'emploi d'une dose test convenable; 2) l'injection lente du produit en plusieurs doses fractionnées et; 3) des vérifications répétées pour ćvitcr l'administration intravasculaire.

Un seul test d'aspiration négatif est inșuffisant et le test doit être répété avant chaque nouvelle injection.

A ce jour, au Canada, on n'a pas rapporté de décès à l'accouchement associć à la bupivacaïne; on a cependant rapporté à Santé et Bien-Etre Canada un décès relié à l'utilisation de bupivacaine à 0.75 pour cent dans le cas du blocage d'un nerf intercostal chez un cas non-obstétrique. Au RoyaumeUni, où tous les décès en couches sont étudiés de façon confidentielle, ${ }^{16}$ on n'a rapporté aucun décès relié à la bupivacaïne à 0.75 pout cent, cependant des décès reliés à une analgésie régionale intraveineuse chez des malades non enceintes ont êté rapportés. ${ }^{17}$ Il semble donc que la corrélation entre l'injecrion intravasculaire de bupivacaine et la toxicité soit réelle mais la corrélation entre la bupivacaine et la mortalité maternelle ne semble se retrouver qu'aux Etats-Unis. Ceci peut être attribuable à la façon d'employer le produit, à la compétence de la personne administrant la drogue, ou encore à quelque autre facteur non encore identifié.

Comme anesthésiste canadien, quelle leçon peuton tirer de cette aventure? La bupivacaïne à 0.75 pour cent qu'on emploie ici depuis 1975 sans incident a été retirée du marché sur la foi de preuves bien minces vepant de l'étranger.

Peut-être le docteur Albright et les organismes gouvernementaux canadiens et américain ont-ils raison, mais dans ce cas-ci, le Canada a perdu une occasion unique d'évaluer scientifiquement la sécurité de l'emploi de la bupivacaïnc à 0.75 pour cent pour une opération en anesthésie obstétricale. Il est évident maintenant qu'aucun comité d'éthique ne donnera le feu vert pour une telle investigation scientjfique. Les médias se sont faits juges et la possibilité 
d'une étude scientifique est maintenant écartée.

Il est temps, peut-être, pour la Société canadienne des anesthésistes de songer aux moyens à prendre pour faire valoir son opinion auprès de Santé et Bien-Etre Canada et des fabricants de produits pharmaceutiques lorsque de tels débats apparaissent. Ceci permettrait aux anesthésistes, à titre d'individus, d'en référer à une filière normale plutôt que de se rêtrouver démunis devant les médias et amenés à émettre des opinions mal assurées facilement manipulables. De plus, chacun devrait faire pression sur Santé et Bien-Etre Canada afin que l'agence suive les conseils de la Société canadienne des anesthésistes plutôt que de laisser des décisions importantes à la fortune du hasard.

\section{References}

1 Albright GA. Cardiac arrest following regional anesthesia with etidocaine or bupivacaine. Anesthesiology 1979; 51: 285-7 (Editorial).

2 Albright $G A$. Maternal mortality with bupivacaine regional anesthesia. 6th European Congress of Anesthesiology 1982: 10 (Abstract).

3 Moore DC, Thampson GE, Crawford RD. Long acting local anaesthetic drugs and convulsions with hypoxia and acidosis. Anesthesiology 1982; 56: 230-2

4 Davis $N L$, deJong $R H$. Successful resuscitation following massive bupivacainc overdose. Anesth Analg 1982; 61: 62-4

5 Block A, Covino BG. Effect of local anesthetic agents on cardiac conduction and contractility. Regional Anesth 1981; 6: 55-61.

6 deJong $R H$, Ronfeld $R A$, deRosa RA. Cardiovascular effects of convulsant and supraconvulsant doses of amide lacal anesthetics. Anesth Analg 1982; 61: 3-9.

7 Kotelko DM, Dailey PA, Brizgys RV, Shnider SM, Levinson $G$. Bupivacaine cardiotoxicity in adult sheep. Anesth Analg 1983; 62: 268 (Abstract).

8 Morishima HO, Pedersen H, Finster M, Feldman $H S$, Covino $B G$. Is etidocaine more cardiotoxic than lidocaine? Anesthesiology 1982; 57: A401 (Abstract).

9 Sage D, Feldman HS, Datta S, Covino B. Influence of lidocaine and bupivacaine on isolated guinea pig atria in the presence of acidosis and hypoxia. Anesthesiology 1982; 57: A197 (Abstract).

10 Thigpen. $W$, Kotelko DM, Shnider SM er al. Bupiva- caine cardiotoxicity in hypoxic-acidotic sheep Anesthesiology 1983; 59: A204 (Abstract).

11 Sage D, Feldman H, Arthur GR, Covino BG. Cardiovascular effects of lidocaine and bupivacaine in the awake dog. Anesthesiology 1983; 59: A210 (Abstract).

12 Liu P, Feldman HS, Covino BM, Giasi R, Covino $B G$. Acute cardiovascular toxicity of intravencus amide local anesthetics in anesthetized ventilated dogs. Anesth Analg 1982; 61: 317-22.

13 Chadwick HS. Relative lidocaine and bupivacaine toxicity in ventilated cats. Anesthesiology 1982: 57: A203 (Abstract).

14 Coyle DE, Denson DD, Thompson GA, Myers JA, Arthur $G R$. Species differences of bupivacaine protein binding. Anesthesiology 1983; 59: A207 (Abstract).

15 Bromage PR. Cardiovascular toxicity of local anesthetics. Irt Epidural Analgesia. Bromage PR. Philadelphia, WB Saunders, 1978: 106-9.

16 Department of Health and Social Security. Report on confidential enquiries into maternal deaths in England and Wales 1973-1975. Her Majesty's Stationary Office, London 1979.

17 Heath $M L$. Deaths after intravenous regional anaesthesia. Br Med J 1982; 285: 913-4. 